\title{
The Study on the Superposition of Mechanical Wave
}

\author{
Jie Wang * \\ School of Electronic and Information Engineering \\ Guangdong polytechnic normal university, \\ Guangzhou, Guangdong, China 510665 \\ e-mail: jane-w03@163.com \\ * Corresponding Author
}

\section{Lina Guo}

School of Electronic and Information Engineering Guangdong polytechnic normal university, Guangzhou, Guangdong, China 510665

guoln523@yahoo.com.cn

\author{
Yinji $\mathrm{He}$ \\ School of Electronic and Information Engineering \\ Guangdong polytechnic normal university, Guangzhou, \\ Guangdong, China 510665 \\ heyingji8@126.com
}

\begin{abstract}
The mechanical wave is emphases and difficult content in physical university course. Using the plane simple harmonic wave as an example, the physical meaning of the wave equation is clarified; the phase difference between the two particles in medium of coherent wave sources is also discussed. Analyzed and simulated the process of the coherent superposition with the software Matlab in this paper to help students to understand and digest this part of content.
\end{abstract}

Keywords- mechanical wave; phase difference; coherent superposition

\section{INTRODUCTION}

A mechanical wave is a disturbance that travels through some material or substance called the medium. As the wave propagates, each portion of the medium exerts a force and does work on the adjoining portion. In this way a wave transports energy and vibration states. Although many characteristics of periodic wave have different propagation mechanism but they have the same regular pattern and can be described by the wave function. Interference is happened on overlapping of waves when they pass through the same region at the same time. The plane simple harmonic wave (transverse wave) is often used as an example to study the characters and laws about mechanical waves. Any complex wave can be analyzed to the overlap of many simple harmonic waves with different frequency or wavelength. The regular pattern of waves is described by wave equations which are also suit for electromagnetic waves, optical waves and De Broglie wave. So it was very important to grasp the physics meaning of simple harmonic wave equations and apply them to solve the problems.

\section{PHYSICS MEANING OF PLANE SIMPLE HARMONIC WAVE FUNCTION}

We will concentrate on sinusoidal waves, in which each particle undergoes simple harmonic motion about its equilibrium position. It turns out that any periodic wave can be represented as a combination of sinusoidal waves.
A wave function is that describes the position of any particle in the medium at any time [1].

Suppose a plane harmonic wave travels in the ideal medium at $x$ direction (which is the wave originates), suppose a certain displace of a particle at the zero point $\mathrm{O}$ $(x=0)$, where the oscillation equation is

$$
y=A \cos (\omega t+\varphi)
$$

the wave speed is given $u$, when the vibration travels to the other particles, which repeat the zero point vibration. Also, let's look at waves on a stretched string, the oscillation phase of particles get behind in turn; the wave propagating in the + or $-\mathrm{x}$ directions, the displacement may be written:

$$
y=A \cos \left[\omega\left(t \mp \frac{x}{u}\right)+\varphi\right]
$$

That is, the particle oscillates in simple harmonic motion with amplitude $A$, frequency $f$, and angular frequency $\omega=2 \pi f$. The notation $y(x=0, t)$ reminds us that the motion of this particle is a special case of the wave function $y(x, t)$ that describes the entire wave. In many university physics teaching materials, suppose that the displacement of a particle at the left end of the string $(x=0)$ is the wave originates. But in many exercises, the wave sources are often not at the zero point ( $x=0)$; that is it would make a mistake if we use the equation above to calculate. So we should amplify on this question.

- The giving particle oscillates is not at the zero point $(\mathrm{x}=0)$; the oscillating equation of the given particle $P$ is:

$$
y_{p}=A \cos (\omega t+\varphi)
$$

and the displacement of $P$ is $x_{0}$, we can deduce the wave function of this mechanical wave (sinus oidal 
wave moving in $+\mathrm{x}$-axis or $-\mathrm{x}$-axis directions) [2,3]:

$$
y=A \cos \left[\omega\left(t \mp \frac{x-x_{0}}{u}\right)+\varphi\right],
$$

where the zero point $\mathrm{O} y_{0}(\mathrm{x}=0, \mathrm{t})$ and point $\mathrm{P} y_{0}$ $(\mathrm{x}=\mathrm{p}, \mathrm{t})$ are at the same wave string or not, the equation are both applied.

- If the zero point $O y_{0}(\mathrm{x}=0, \mathrm{t})$ and point $P y_{0}(\mathrm{x}=$ $\mathrm{p}, \mathrm{t})$ are not at the same wave string shown as Fig. 1 , supposed point $\mathrm{A}$ as the wave source, the given paticle $P$ is the beginning point, the oscillation function is $y_{p}=A \cos (\omega t+\varphi)$, we can obtain the wave function :

$$
y=A \cos \left[\omega\left(t \mp \frac{x-x_{p}}{u}\right)+\varphi\right]
$$

Where the arrangement of the function must be payed attention to: in (4), only $x \geq x_{A}$, the formula has physics meaning at $+\mathrm{x}$ directon; on the other hand, only $x \leq x_{A}$, the formula has physics meaning at $-\mathrm{x}$ direction.

$\underset{O}{x_{A}} \quad$\begin{tabular}{c}
$x_{p}$ \\
\hline
\end{tabular}

Figure 1. Graph of the displacement of $\mathrm{O}(\mathrm{x}=0)$ and $\mathrm{P}$ (the beginning point)

\section{PHASE DIFFERENCE OF T WO POINTS}

A mechanical wave propagates the state of vibration in the medium. Phase different is a very important physical quantity which plays the role of an angular quantity (always measured in radians)[4-6], and its value determines what part of the sinusoidal cycle is occurring at a particular point and time. Suppose there are two points $x_{1}$ and $x_{2}$ in one mechanical wave string at the same time; the phase of them are:

$$
\begin{gathered}
\varphi_{1}=w t-\frac{\omega x_{1}}{u}+\varphi=w t-\frac{2 \pi x_{1}}{\lambda}+\varphi \\
\varphi_{2}=w t-\frac{2 \pi x_{2}}{\lambda}+\varphi .
\end{gathered}
$$

The phase difference of them are :

$$
\Delta \varphi=\varphi_{2}-\varphi_{1}=\frac{-2 \pi}{\lambda}\left(x_{2}-x_{1}\right)=\frac{-2 \pi}{\lambda} \Delta x \quad(5) .
$$

We can obtain the relationship of $\Delta \varphi$ (the phase difference of abitrary two points in the wave string) and $\Delta x$ (the difference of wave distance) which reflect the two oscillate ragged in some extent. If two points are not on a string in the medium, what is the phase difference of them? For example, a plane harmonic wave travels along the $+x$ axis, wavelength is $\lambda$,frequncy is $f$, and wave source is at the point $S_{o}$ shown as Fig. 2.

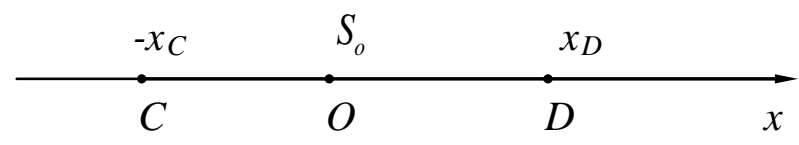

Figure 2. Graph of the arbitrary two points $\mathrm{C}$ and $\mathrm{D}$ (which are not on the same wave string)

Suppose the displacement of wave source $S_{o}$ is the zero point $O(\mathrm{x}=0), C\left(\mathrm{x}=-x_{C}\right)$ and $D\left(\mathrm{x}=x_{D}\right)$. It would make a mistake if we use the (5) to caculate the phase difference of point $C$ and point $D$. So we can caculate $\Delta \varphi_{D O}$ (the phase difference of $D$ and $O$ ) and $\Delta \varphi_{C O}$ (the phase difference of $C$ and $O$ ) first.

$$
\Delta \varphi_{D O}=\varphi_{D}-\varphi_{O}=-\frac{2 \pi}{\lambda} x_{D}
$$

$$
\Delta \varphi_{C O}=\varphi_{C}-\varphi_{O}=-\frac{2 \pi}{\lambda} x_{C}
$$

Then the phase difference of $C$ and $D \Delta \varphi_{C D}$ is :

$$
\Delta \varphi_{C D}=\varphi_{C}-\varphi_{D}=\Delta \varphi_{C O}-\Delta \varphi_{D O}=\frac{2 \pi}{\lambda}\left(x_{D}-x_{C}\right)
$$

From the example above, the displacement of the wave source, beginning point and the direction of wave string which affect the wave function and the state of particles vibration greatly.

\section{PHASE DIFFERENCE AND AMPLITUDE OF SUPERPOSITION}

In a region where wave-trains from coherent sources cross, superposition occurs giving reinforcement of the waves at some points and cancellation at others. Suppose there are two coherent wave sources; what is the phase difference of the particles in the region of they meeting. For example, $S_{1}$ and $S_{2}$ are two coherent wave sources with the same amplitude $A_{1}$ and wavelength $\lambda$; the 
distance of $S_{1}$ to $S_{2}$ is $\lambda / 4$, the phase of $S_{1}$ is advance to $S_{2} \pi / 2$, shown as Fig. 3

$\longrightarrow S_{1} \quad S_{2}$

Figure 3. Graph of two coherent wave sources $S_{1} S_{2}$

Coherent sources $S_{1} S_{2}$ have a constant phase differece which means they must have the same frequency and for complete cancellation to occure the amplitudes of the superposing waves they produce must be about equal. Suppose the displacement of wave source $S_{1}$ is the point ( $\mathrm{x}=0$ ); $S_{1} S_{2}$ travel along the $\mathrm{x}$-axis and they can traveling at the positive and negative direction of $\mathrm{x}$-axis at the same time.

The oscillation function of $S_{1}$ is :

$$
A_{1} \cos (\omega t+\varphi+\pi / 2)
$$

thanks to foregone the phase of $S_{1}$ is advance to $S_{2}$ $\pi / 2$. The wave functions of $S_{1}$ traveling to the $+\mathrm{x}$-axis are:

$$
y_{1}(x, t)=A_{1} \cos [\omega(t-x / u)+\varphi+\pi / 2)(x \geq 0),
$$

The wave functions of $S_{1}$ traveling to the - x-axis are:

$$
y_{1}(x, t)=A_{1} \cos [\omega(t+x / u)+\varphi+\pi / 2)(x \leq 0),
$$

In the same theory, the wave functon of $S_{2}$ traveling to the $+\mathrm{x}$-axis are:

$$
y_{2}(x, t)=A_{1} \cos [\omega(t-(x-\lambda / 4) / u)+\varphi)(x \geq \lambda / 4)
$$

The wave functon of $S_{2}$ traveling to the - x-axis are:

$$
y_{2}(x, t)=A_{1} \cos [\omega(t+(x-\lambda / 4) / u)+\varphi)(x \leq \lambda / 4)
$$

We can divide three regions to discuss which the phase difference of partic les in the region of two sources meeting.

- In the region I : $x \leq 0, S_{1} S_{2}$ are both moving in the - $\mathrm{x}$-axis , the wave function (8) and wave function ( 10$)$ are satisfied, owing to $\omega / u=2 \pi / \lambda$, so the phase difference of two waves is:

$$
\Delta \varphi=\frac{\pi}{2}+\frac{2 \pi}{\lambda}\left(x-\left(x-\frac{\lambda}{4}\right)\right)=\pi
$$

$S_{1} S_{2}$ are cancellation and

$$
y_{1}(x, t)+y_{2}(x, t)=0
$$

and the amplitude

$$
A=A_{1}-A_{1}=0
$$

- In the region II : $0 \leq x \leq \lambda / 4, S_{1}$ are moving in the +x-axis, $S_{2}$ are taveling in the - x-axis, the wave function (7) and wave function (10) are satisfied, so the phase difference of two waves:

$\Delta \varphi=\frac{\pi}{2}-\frac{2 \pi}{\lambda}\left(x+\left(x-\frac{\lambda}{4}\right)\right)=\frac{4 \pi x}{\lambda}+\pi$

$S_{1} S_{2}$ are reinforcement and the amplitude $A$ (overlaping of $S_{1} S_{2}$ ) has a relationship to the displacement $x$; and the amplitude $A$ are:

$A=\sqrt{A_{1 x}^{2}+A_{2 x}^{2}+2 A_{1 x} A_{2 x} \cos \Delta \varphi}$
$=\sqrt{A_{1 x}^{2}+A_{2 x}^{2}-2 A_{1 x} A_{2 x} \cos \frac{4 \pi x}{\lambda}}$

- In the region III: $x \geq \lambda / 4, S_{1} S_{2}$ are moving in the $+\mathrm{x}$ axis, the wave function (7) and wave function (9) are satisfied, so the phase difference of two waves:

$$
\Delta \varphi=\frac{\pi}{2}-\frac{2 \pi}{\lambda}\left(x-\left(x-\frac{\lambda}{4}\right)\right)=0 .
$$

$S_{1} S_{2}$ are interference and the amplitude $A$ of overlaping are :

$$
A=A_{1}+A_{1}=2 A_{1} .
$$


In order to better illustrate this problem, we need one sorfware to draw some figures . MATLAB is good choice; it is a high-level technical computing language and interactive environment for algorithm development, data visualization, data analysis, and numeric computation. Using MATLAB, you can solve technical computing problems faster than with traditional programming languages, such as $\mathrm{C}, \mathrm{C}++$, and Fortran... MATLAB provides a number of features for documenting and sharing your work. You can integrate your MATLAB code with other languages and applications, and distribute your MATLAB algorithms and applications. It help you develop and enhance curriculum, facilitate lectures and classroom examples, and inspire student learning.

We use the software Matlab to draw the curve of three region of $S_{1} S_{2}$ overlaping. It is applied in Fig. 4. The three regions in the graph of $S_{1} S_{2}$ is interference; in region I the amplitude $\mathrm{A}$ is 0 ; in region II the amplitude $\mathrm{A}$ is different from the displacement $\mathrm{x}$; in region III the amplitude $\mathrm{A}$ is double.

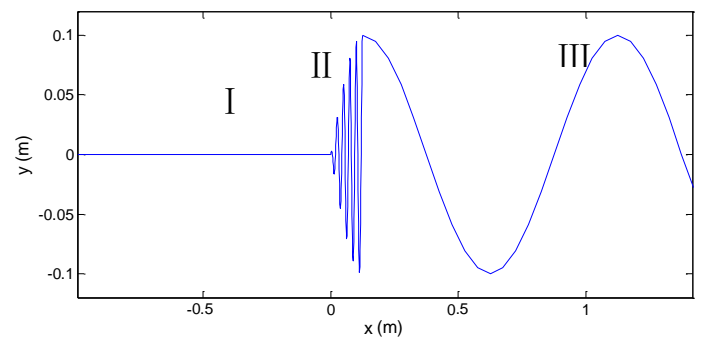

Figure 4. Graph of two coherent wave $S_{1} S_{2}$ interference

Usually the students divide these questions into two regions simply when they analyze them.

- Outside of source $S_{1}$, the particles with the distance $r_{1}$ to the $S_{1}$, the phase difference of $S_{1} S_{2}$ traveling to this point is :

$$
\Delta \phi=\frac{\pi}{2}-\frac{2 \pi}{\lambda}\left[r_{1}-\left(r_{1}+\frac{\lambda}{4}\right)\right]=\pi
$$

The total amplitude A and intensity I are:

$$
\begin{gathered}
A=A_{1}-A_{1}=0 \\
I=A^{2}=0
\end{gathered}
$$

- Outside of source $S_{2}$, the particles with the distance $r_{1}$ to the $S_{2}$, the phase difference of

$$
\begin{aligned}
& S_{1} S_{2} \quad \text { traveling to this point is: } \\
& \Delta \phi=\frac{\pi}{2}-\frac{2 \pi}{\lambda}\left(r_{2}+\frac{\lambda}{4}-r_{2}\right)=0
\end{aligned}
$$

The total amplitude $A$ and intensity $I$ are:

$$
\begin{gathered}
A=A_{1}+A_{1}=2 A_{1} \\
I=A^{2}=4 A_{1}^{2}
\end{gathered}
$$

But in the region between $S_{1} S_{2}$, they are often ignored. Even some student noticed this region between $S_{1} S_{2}$, they don't know how to analyze or don't know what tools to analyze. Now there are some questions need to be explained:

- the amplitude $A$ of mechanical wave is a function of time $t$ and displacement $x$, so the two wavetrains cross the total displacement is the vector sum of the individual displacement due to each wave at that point. In the region III (also outside of source $S_{2}$ ), the vibration phase different of any point is 0 when $S_{1} S_{2}$ travels to the point; $S_{1} S_{2}$ is interference reinforcement; the total amplitude is constant $2 A_{1}$, but the distance to $\mathrm{x}$-axis is not $2 A_{1}$. It is a sinusoid can be known from Fig. 4 . Owing to the two sources have the same amplitude $A_{1}$, the total amplitude is double in a whole.

- the intensity $I$ of the wave may be defined to be the mean-square value of displaement (a quantity proportional to energy density): $I=\overline{A^{2}}$, but it can not be say the wave intensity $I$ is equal to the square of the amplitude $A^{2}$; so the students figure $S_{1} S_{2}$ in the interference reinforcement $I=A^{2}=4 A_{1}^{2}$ is not accurate; it is needed some Prerequisite.

\section{CONCLUSIONS}

Basing on the physical meaning of the plane harmonic wave function, it was analyzed that the phase difference of arbitrary two points when wave sources pass through different regions in the medium. It aimed to understand the phase difference, amplitude and wave intensity of harmonic waves when they are meeting and interference. We analyzed and simulated the different sources and draw the graphs with the software Matlab in order to help students understand digest the wave interference and superposition.

\section{ACKNOWLEDGMENT}

The paper is supported by the science research fund of Guangdong Polytechnic Normal University (13KJY07). 


\section{REFERENCES}

[1] Kenneth W. Ford, Classical and Modern Physics Volume 2, a textbook for students of science and engineering. Xerox college publishing, Lexington, pp. 887-947,1974

[2] T. Duncan BSC, Physics, a textbook for advanced level students. John Murray publishers Ltd. London, pp.339-362,1984

[3] Marcelo Alonso, Edward J. Finn, Fundamental University Physics 2nd Edition, Volume Two Fields and waves, Addison-Wesley Publishing Company Inc. London, pp. 505-512, 1983

[4] M. Nelkon M S C., F. Inst. P., A.K.C., Principles of Physics sixth Edition with SI Units, Granada Publishing Ltd., Hertfordshire, pp.351-381,1977

[5] Sears and Zemensky's University Phisics with modern Physics (11th Ed) Adapted by Deng Tie-ru etc. China Machine Press, Beijing, pp 320-340,2009

[6] Jia-qing Li , Jin Chen, Chao Yang, Wave superposition based sound field reconstruction. Journal of Shanghai Jiaotong University (Science), pp 381-384, 2008
[7] BI Chuanxing,HU Dingyu,XU Liang,ZHANG Yongbin. Recovery of the free field in noisy environment by using the spherical wave superposition method[J]. Chinese Journal of Acoustics, pp 42-53, 2014.

[8] Multi-directional Wave Superposition of Wave Diffraction [J]. China Ocean Engineering, pp 165-175, 1989.

[9] D. J. Han *, J. J. Luo Department of Civil Engineering, South China University of Technology, Guangzhou, 510640 China. Simulation of Stochastic Fluctuating Wind Field Using the Wave Superposition Method with Random Frequencies[A].2006.

[10] Comparison study on spherical wave superposition method and spherical wave source boundary point method for realizing nearfield acoust ic holography[J]. Chinese Science Bulletin, pp 472484, 2005.

[11] Near-Field Acoustic Holography for Semi-Free Acoustic Field Based on Wave Superposition Approach [J]. Chinese Journal of Mechanical Engineering, pp 455-459, 2006. 